\title{
KINEMATIC PREDICTION OF INTERSEGMENT LOADS AND POWER AT THE JOINTS OF THE LEG IN WALKING*
}

\author{
M. Y. ZARRUGH \\ Department of Mechanical Engineering and Applied Mechanics, \\ University of Michigan, Ann Arbor, MI 48109, U.S.A.
}

\begin{abstract}
The left and right ground reactions are predicted from computed absolute motion data with the aid of some simple assumptions regarding symmetry between the forward shear forces during the double support phase and the rate at which support is transferred from one leg to the other. The computed ground forces are then used to evaluate forces, moments and power at the joints of the lower limb. The power supplied at the joints of the lower limbs is found to agree well with the power required to increase the energy level of the body.
\end{abstract}

\section{INTRODUCTION}

Energy and power requirements can be treated analytically from two points of view: the first focuses on the changes in mechanical energy of segments and the second on the work done at the joints. The first point of view was treated in Zarrugh (1981). The second point of view is considered in this paper in which the power supplied for walking is evaluated as the rate of work done at the joints. This rate of work or power is the product of the moment and the relative angular velocity at each joint. If all forces, including ground reactions, are known, the moment at each joint can be determined. The ground reactions can either be measured directly using a force plate or computed indirectly from kinematic data. The former approach is less time consuming and more accurate than the latter. However, kinematic prediction is adequate, if usage of the force plate is not feasible and the inherent inaccuracy is tolerated

The prediction of ground reactions from measured motions is relatively new because of the recent availability of a fast means of recording and analyzing motion data. If the acceleration of each segment is known, the acceleration of the total body and hence the total ground force can be computed. This total ground force cannot be divided into its left and right components without knowing how body support is shared between the legs.

The first attempt to predict joint moments was made by Fischer (1906) who determined the moments at the joints of the lower limb during swing phase (i.e. single support phase). Foot pressure measurements allowed Elftman $(1939,1940)$ to remove the indeterminate nature of the double support phase and to predict the sagittal plane forces and moments in the leg during walking and running. With the development of the

* Received 5 September 1979; in revised form 18 June 1981. force plate (Cunningham, 1950; Cunningham and Brown, 1952), many studies appeared predicting joint loads to aid in the design of orthopedic implants or in the evaluation of joint therapy (Bresler and Frankel, 1950; Cappozo et al., 1975; McGrouther, 1974). In such studies, some authors have emphasized particular skeletal joints: the hip (Paul, 1967) and the knee (Morrison, 1970).

Prediction of the ground forces from kinematic measurements has been attempted in a few studies (Thorton-Trump and Daher, 1974; Abdelnour et al., 1975), but neither of these studies reported reactions that were in good agreement with force plate records or adequately described the assumptions made to remove the indeterminate condition present in the double support phase.

\section{METHODS}

The purpose of this paper is to compute the ground forces from absolute kinematic data that are in turn computed from relative motion measurements as described in Zarrugh and Radcliffe (1979) for several walking speeds ranging from 0.9 to $2.2 \mathrm{~m} / \mathrm{s}$ on a level treadmill at step rates chosen by the subject (freewalking). The results of one typical subject who is well accustomed to treadmill walking are reported.

For the purpose of the analysis, the body is divided into seven rigid components: HAT (Head-ArmsTrunk) and three segments, thigh, shank and foot, for each of the lower limbs. The absolute motion of each of these divisions was computed from displacements measured using string transducers and selfaligning electro-goniometers. Three pairs of strings were used to observe the absolute position of the HAT in space. Relative rotations at the joints of the right limb were measured using three sets of threedimensional goniometers at the hip, knee and ankle. The kinematic data, as well as the displacement of the 
treadmill, were gathered at a rate of 200 samples/s for 32 steps at each step rate used. Data were averaged to obtain mean values of all measurements. Prior to any further reduction, data were filtered by harmonic truncation at the twelfth harmonic. The error associated with reduced measured data was not expected to exceed $6 \%$.

The mixed set of absolute and relative measurements were converted into absolute three-dimensional motion data of the mass centers as detailed in Zarrugh and Radcliffe (1979). A numerical harmonic differention scheme was used to compute the first and second derivatives of the displacement data. Only the lower 7 to 12 harmonics are taken into account. The rest are discarded before converting displacement coefficients into derivative coefficients. A justification of this filtering technique by harmonic truncation appeared in Zarrugh and Radcliffe (1979).

\section{MATHEMATICAL FORMULATION}

\section{Equation of motion of the body}

If the motion of the center of mass of each segment is known, the motion of the body as a whole is described by

$$
\mathbf{F}_{\mathrm{R}}+\mathrm{F}_{\mathrm{L}}=\sum_{i} m_{i}\left(\ddot{\mathbf{c}}_{i}-g\right)
$$

where

$F_{\mathrm{R}}=$ right ground reaction,

$\mathbf{R}_{\mathbf{L}}=$ left ground reaction,

$m_{i}=$ mass of segment $i$,

$\ddot{c}_{i}=$ acceleration of the center of mass of segment $i$,

$g=$ acceleration due to gravity.

If the ground reaction moment is neglected (this is not valid at low speeds; see Cunningham, 1950), the body moment balance is as follows:

$$
\begin{aligned}
\mathbf{a}_{\mathrm{R}} \times \mathbf{F}_{\mathrm{R}}+\mathbf{a}_{\mathrm{L}} \times \mathbf{F}_{\mathrm{L}}=\sum_{i}\left[I_{i}\right] \ddot{\boldsymbol{\theta}} \\
+\sum_{i} m_{i} \mathbf{c}_{i} \times\left(\ddot{\mathbf{c}}_{i}-\boldsymbol{g}\right),
\end{aligned}
$$

where

$a_{R}=$ Coordinates of the right center of pressure,

$\mathbf{a}_{\mathbf{L}}=$ Coordinates of the left center of pressure,

$\left[I_{i}\right]=$ moment of inertia matrix of segment $i$ about the fixed coordinate axes,

$\ddot{\theta}_{i}=$ absolute angular acceleration of segment $i$,

$c_{i}=$ center of mass of segment $i$.

During single support phase when only one ground force is present, both the force and its center of pressure can be found from the simultaneous solution of equations (1) and (2). However, during double support phase, these equations are not linearly independent and hence cannot be used to solve the ground reactions even if their points of application are known. The linear dependence exists because the centers of pressure lie on the same plane which eliminates the scalar equation describing the moment balance about a vertical axis. Therefore, some assumptions must be made regarding the center of pressure during double support. In addition, the assumption of "symmetry" between the fore-and-aft components of the right and left foot will be used to compute the ground reactions in double support phase.

\section{Center of pressure}

The following assumptions are made in order to compute the centers of pressure:

(1) Level walking.

(2) During stance phase, the center of pressure on each shoe moves linearly forward, with time, along a horizontal line parallel to the plane of progression.

(3) Transfer of support from one foot to the other occurs at a variable rate.

(4) Symmetrical fore-and-aft shear forces during double support.

The first assumption means that at any time during the walking cycle, the vertical coordinates of the right, left and total centers of pressure equal zero. The second expresses the horizontal coordinates of the center of pressure along each foot as the sum of the horizontal coordinates of the heel at the instant of contact with the ground plus the portion of the shoe length that rolls on the floor at a steady rate. The same assumption implies constant tread width. Hence, the lateral coordinate of the center of pressure under each foot is the same from heel contact to toe-off. The third assumption indicates that the common center of pressure moves slowly soon after one foot contacts the ground or when the other prepares to lift off as depicted in Fig. 1. The fourth assumption of fore-andaft shear symmetry can be expressed analytically as follows:

$$
\begin{aligned}
& \mathbf{F}_{\mathrm{R} x}(t)=-\mathbf{F}_{\mathrm{Lx}}\left(t_{\mathrm{DS}}-t\right) \\
& \text { and } \\
& \mathbf{F}_{\mathrm{Lx}}(t)=-\mathbf{F}_{\mathrm{R} x}\left(t_{\mathrm{DS}}-t\right) \\
& \text { where }
\end{aligned}
$$

$F_{R x}=$ forward component of the right ground force,

$F_{L x}=$ forward component of the left ground force, $t=$ time in percent of cycle,

$t_{D S}=$ time marking the end of double support. The expressions are true for any time during double support phase. When the equations (3) are combined, they reduce to a simple statement that the algebraic difference between the two shear forces is constant. The above assumptions allow the determination of both right and left centers of pressure, as well as give an additional scalar equation for the horizontal shear force required to solve for the ground reactions.

\section{Joint loads and power}

Figure 2 represents schematically an idealized twojoint segment of the lower limb. The inertia and gravity forces are lumped into one effective force and rotatory inertia is shown as an effective couple, so that d'Alembert's method can be used. The loads in the proximal joint $a_{i}$ can be found through the loads of the distal joint $a_{i+1}$ which are known at this point in 


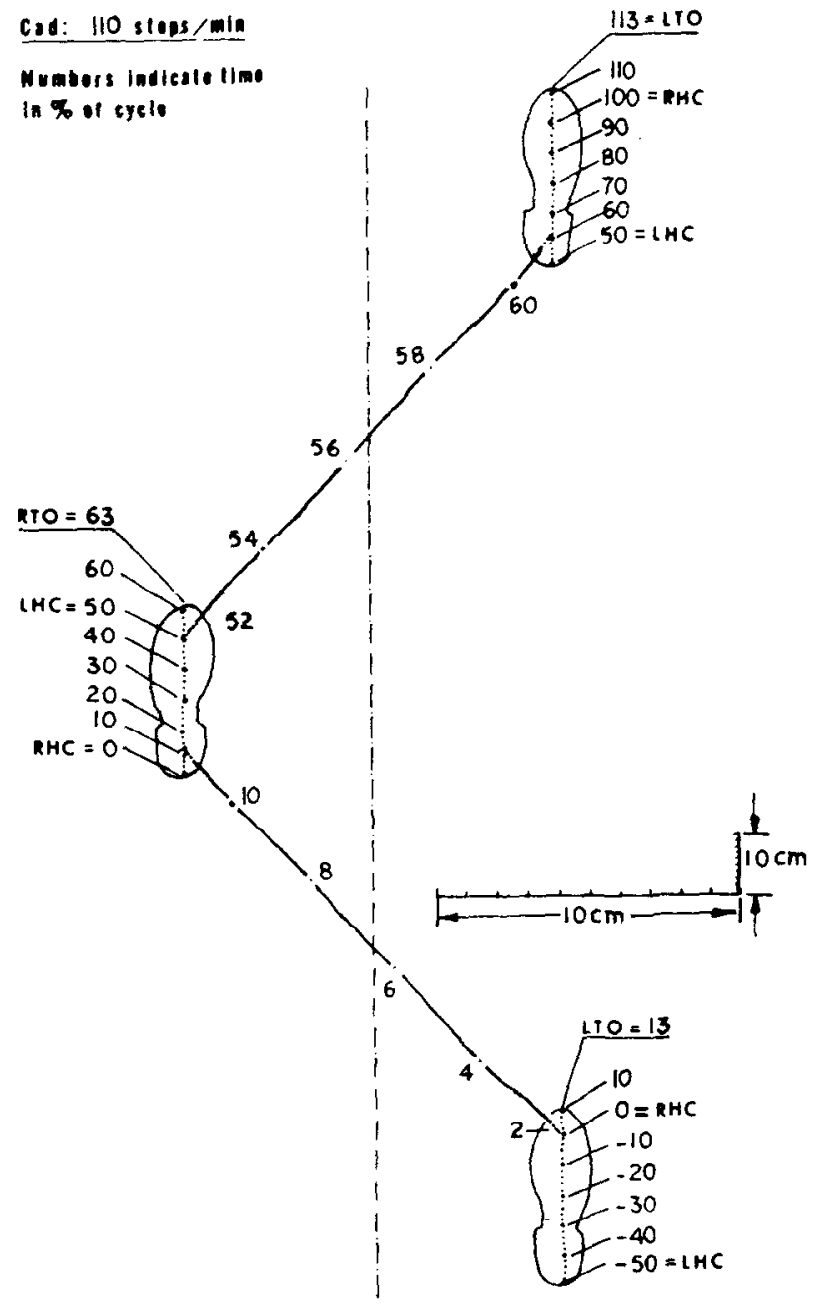

Fig. 1. Motion of the common center of pressure on the floor at a representative step rate of $110 \mathrm{steps} / \mathrm{min}$ corresponding to a speed of $1.5 \mathrm{~m} / \mathrm{s}$.

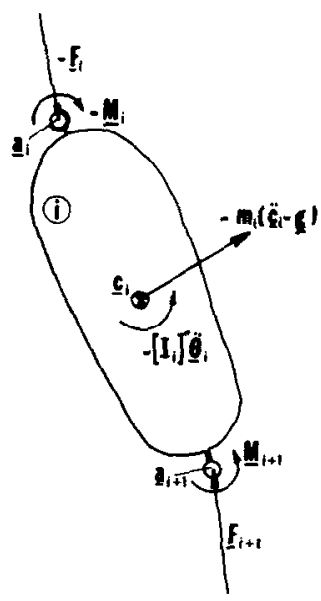

Fig. 2. The free-body diagram of an idealized two-joint segment of the lower limb. 
analysis since the analysis starts at the foot and proceeds to the more proximal segments. The force and moment in the proximal joint are given by

$$
\begin{aligned}
\mathbf{F}_{i} & =\mathbf{F}_{i+1}-m_{i}\left(\ddot{\mathbf{c}}_{i}-g\right), \\
\mathbf{M}_{i} & =\left(\mathbf{a}_{i+1}-\mathbf{c}_{i}\right) \times \mathbf{F}_{i+1}-\left[I_{i}\right] \ddot{\theta}_{i} \\
& -\left(\mathbf{a}_{i}-\mathbf{c}_{i}\right) \times \mathbf{F}_{i}+\mathbf{M}_{i+1}
\end{aligned}
$$

where

$$
\begin{aligned}
\mathbf{F}_{i}, \mathbf{M}_{i}= & \text { force and moment in the proximal joint } \\
& \text { of segment } i
\end{aligned}
$$

$\mathbf{F}_{i+1}, \mathbf{M}_{i+1}=$ loads in the distal joint of segment $i$

$$
\mathbf{c}_{i}=\text { center of mass of segment } i
$$

The sense of moments follows the right hand rule. A positive moment in a joint produces a positive angular acceleration in the segment proximal to it. The same moment produces a negative angular acceleration in the segment located distally. The power input to the proximal member $i-1$ at joint $a_{i}$ is the dot product of moment $\mathbf{M}_{i}$ with the angular velocity of the segment above it, $\mathbf{M}_{i}, \dot{\theta}_{i-1}$. While the input to the distal member, $i$, is $-M_{i} . \theta_{i}$. Therefore, the power, output of joint $i$ is

$$
\dot{W}_{i}=-\mathbf{M}_{i} \cdot\left(\dot{\theta}_{i}-\dot{\theta}_{i-1}\right)
$$

\section{RESULTS AND DISCUSSION}

\section{Predicted ground reactions}

The computed right and left ground reactions are shown in Figs. 3-5. In Fig. 3 the fore-and-aft shear force is displayed as a function of step rate. A positive fore-and-aft shear force is applied to the foot by the ground in a forward direction. Both left and right reactions increase in amplitude with cadence. Although these shear forces, at low cadences, compare well with published force plate data e.g. Cunningham 1950, Figs. 1-12), the computed right shear exhibits a feature not shown in typical force plate records at high cadence. This feature indicates a backward push of the ground on the right foot prior to toe-off $(50-63 \%$ of cycle). The discrepancy may be due to the assumption of equal left and right step durations or due to the digital differentiation scheme employed.

Figure 4 depicts the computed vertical ground reactions on both right and left legs. Body weight in Newtons is shown as a dashed line. In agreement with Cunningham (1950), the inertia effects become increasingly more pronounced as cadence (and speed) increases. At a cadence of $90 \mathrm{steps} / \mathrm{min}$, the vertical force slowly varies about the body weight during single support phase (Fig. 4a). As cadence increases, the maximal vertical force increases and reaches a level twice as large as body weight at $130 \mathrm{steps} / \mathrm{min}$, corresponding to a speed of $1.85 \mathrm{~m} / \mathrm{s}$. The minimal vertical force is also influenced by speed; it decreases with cadence to about $20 \%$ of body weight at 130 steps/min.

Left and right lateral floor reactions are displayed in Figs. $5 a$ and $5 b$, respectively. A positive lateral shear

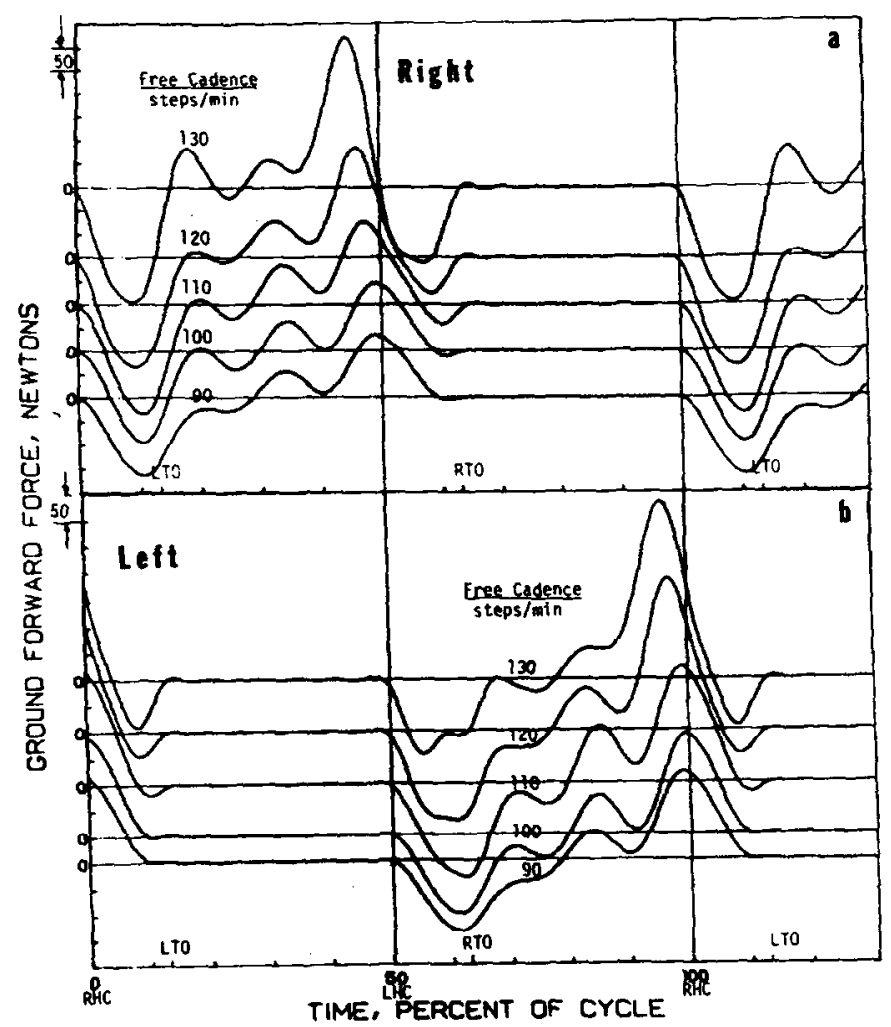

Fig. 3. Forward component of the prodicted ground forces acting on the feet. For this subject, the walking speed in $\mathrm{m} / \mathrm{s}$ equals the cadence squared in (steps/min) $)^{2}$ divided by 8000 . 


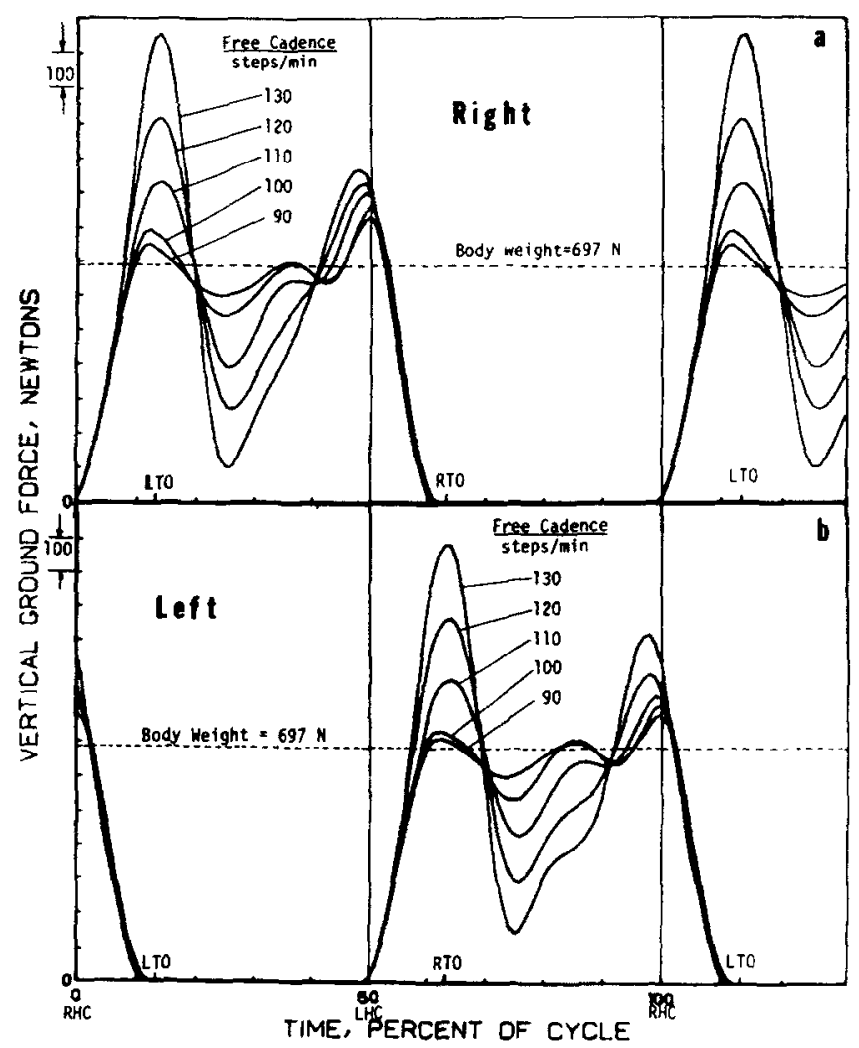

Fig. 4. Vertical component of the predicted ground forces acting on the feet.

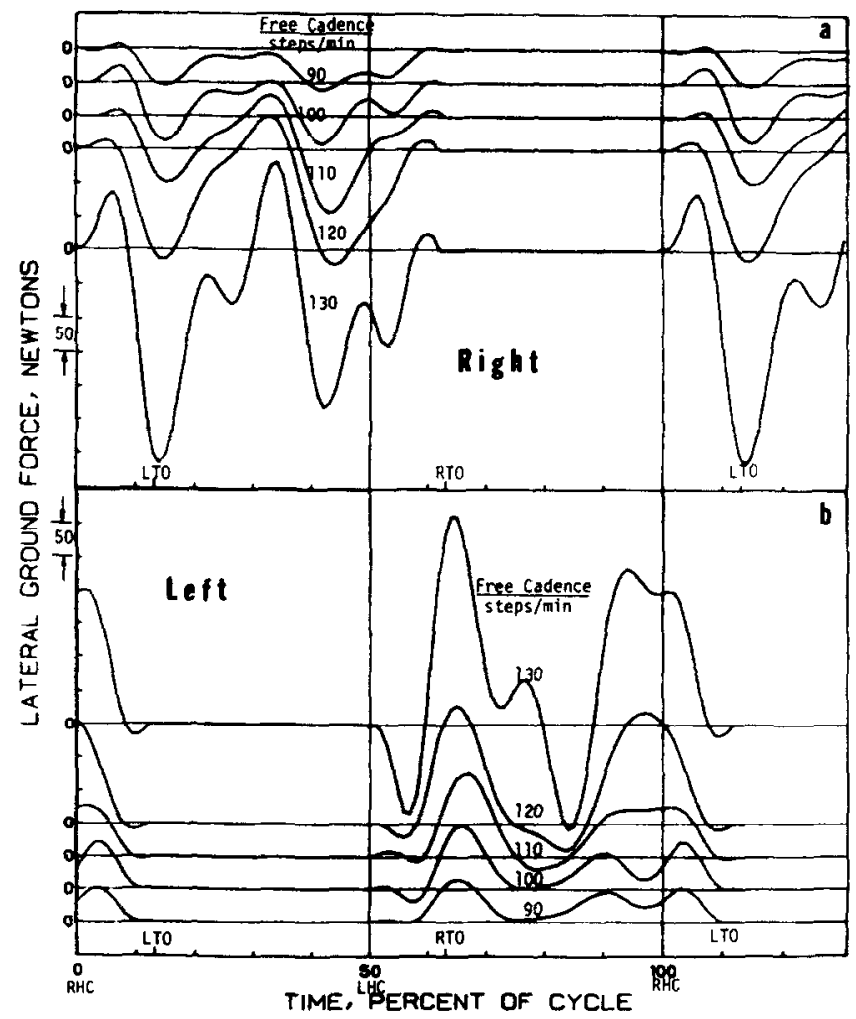

Fig. 5. Lateral component of the predicted ground forces acting on the feet. 
force represents a push by the ground on the subject directed from his left to right. At the cadence of 90 steps/min, the extremes of these forces are about $50 \mathrm{~N}$ or $8 \%$ body weight. These extreme values increase rapidly with cadence to about $40 \%$ of body weight at $130 \mathrm{steps} / \mathrm{min}$. The shapes of the predicted curves in Fig, 5 are similar to those obtained in force plate measurements, but by comparison the value of the extremes is high.

\section{Intersegment forces}

Forces at the joints of the lower limb are computed from the predicted ground reactions and segment motions (Zarrugh and Radcliffe, 1979). Components of these forces along a horizontal forward axis are shown in Fig. 6. Their course with time follows closely those of the fore-and-aft shear forces of the ground indicating that the ground reactions are the main contributors to the forces in the joints during stance phase. Because of the increasing effect of muscle moments, gravity and inertia forces, the joint forces become less and less similar to the corresponding ground forces the higher the joint is from the foot. In swing phase $(63-100 \%)$, the forces at the joints, unlike those of the floor, do not equal zero. The forces at all three joints (Fig. 6) indicate that a horizontal positive force is applied by each segment on the segment proximal to it during the latter part of swing phase $(85-100 \%)$

Vertical forces at the ankle, knee and hip are shown in Fig. 7. Their shape is similar to the vertical ground reactions, except during much of swing phase when negative vertical forces exist at each joint due to the weight and inertia of the limb segments below it.

Figure 8 displays the lateral shear forces in the joints of the right lower limb. Again, these forces are similar in shape to the lateral floor reactions. The hip has a larger lateral force than other joints during swing

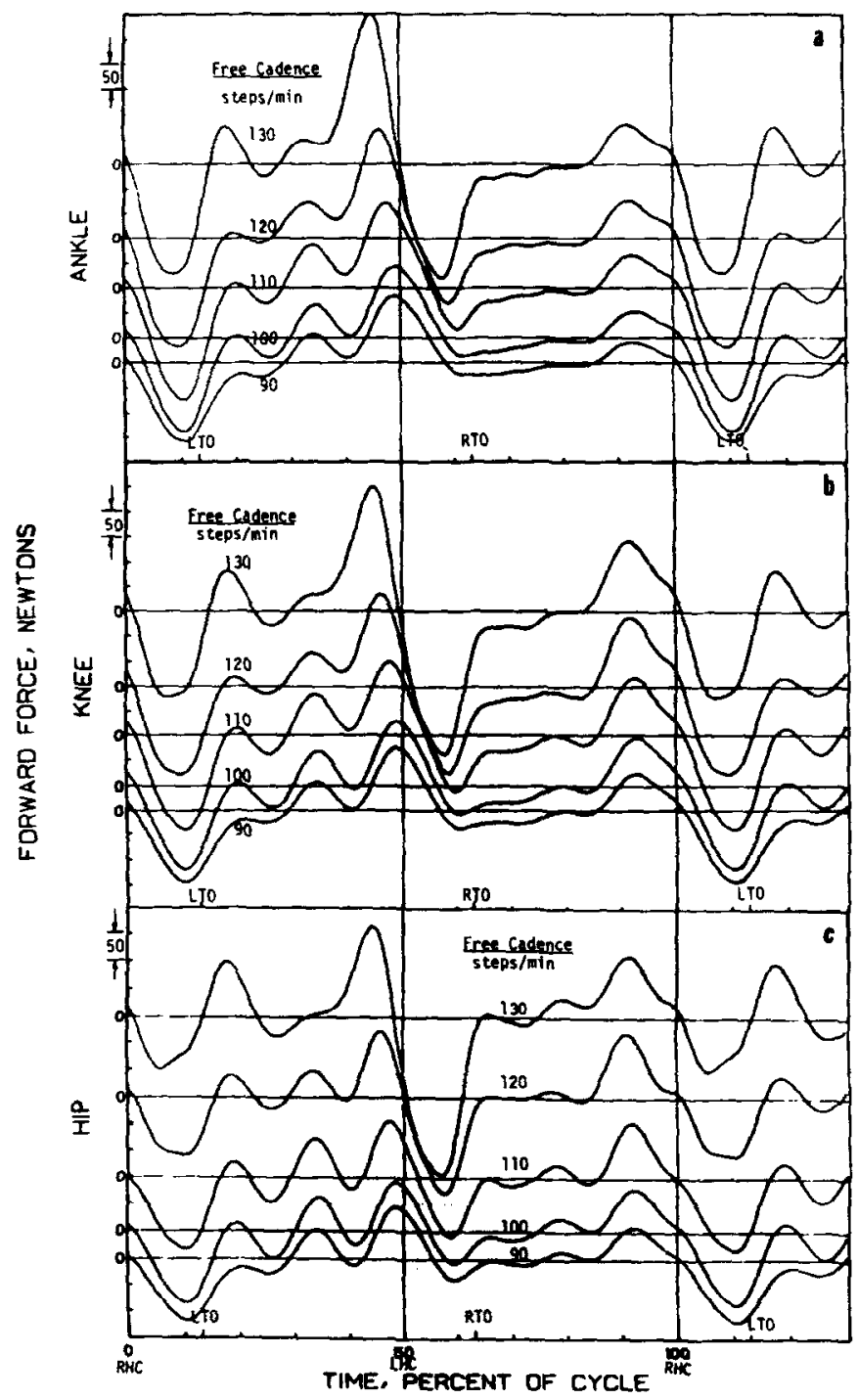

Fig. 6. Forward components of joint forces in the right leg. 


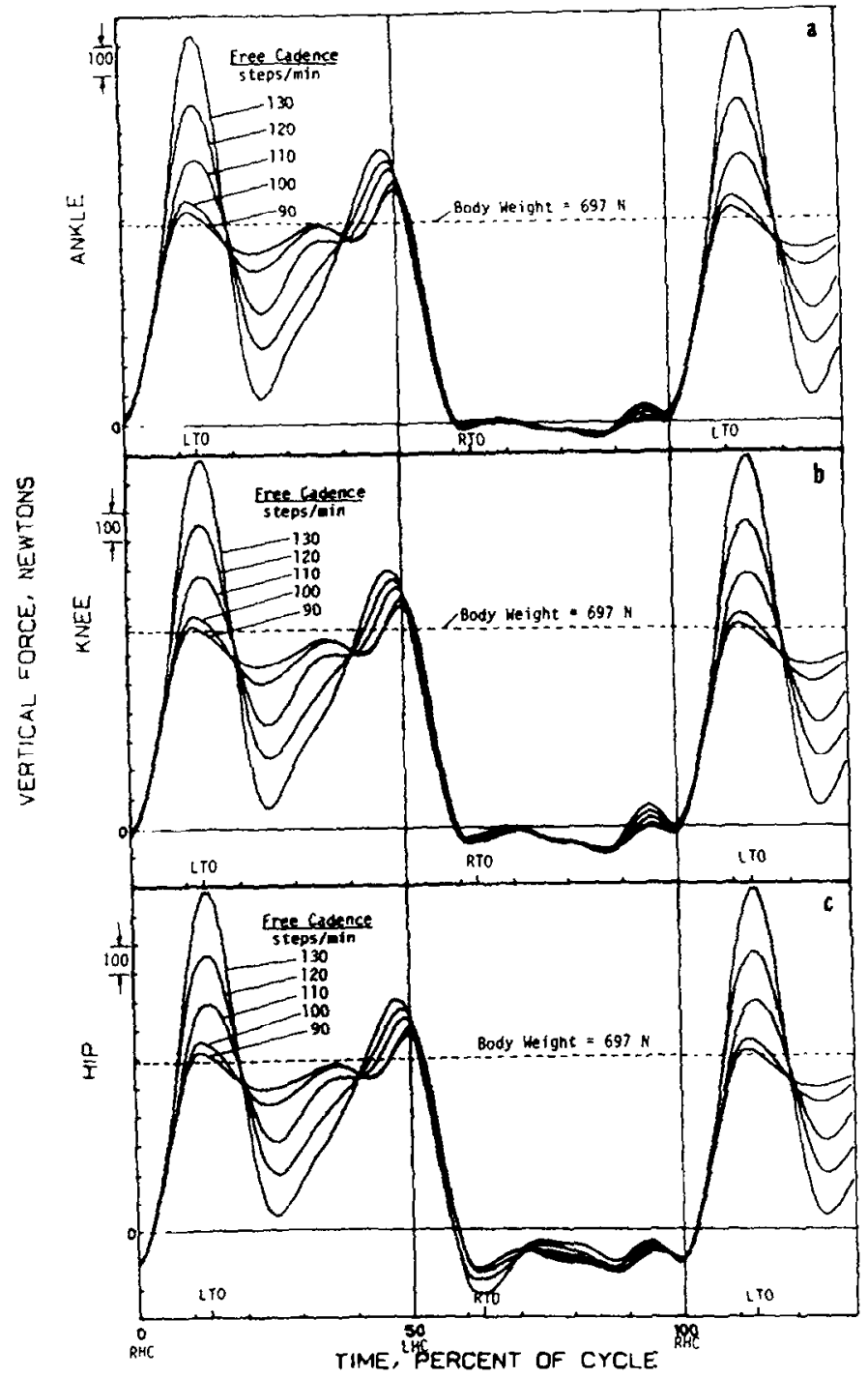

Fig. 7. Vertical components of joint forces in the right leg.

phase at all cadences, while, during stance phase, it tends to have the smallest lateral force. In conclusion, the ground reactions are the main determinants of the forces in the joints during stance phase. In swing phase, the effects of gravity, muscular moments and inertia become stronger the more proximal is the joint.

\section{Joint moments}

Moments in the joints about a horizontal forward axis are shown in Fig. 9. A positive moment at a joint imparts positive angular acceleration to the proximal segment and negative angular acceleration to the distal segment. This action, when viewed in the sagittal plane, tends to assist flexion and inhibit extension at the joint. The shape of the moment curves displayed in Fig. 9 resemble those of the lateral shear force of the ground (Fig. 5) since this force is the major contributor to this moment. Because the hip is the highest joint above the floor, it is shown in Fig. 9 to have the largest moment at any cadence. Similar results were computed by Bresler and Frankel (1950) from force plate measurements.

Because the ground forces have small moment arms about a vertical axis, the moments at the joints about a vertical axis are extremely small compared to other components. These are displayed in Fig. 10. The shape of each moment curve is similar to the corresponding forward shear force of the ground, because this force is much larger than lateral shear force which is the other contributor to this moment. The effect of the ground force does not increase with joint elevation above the floor, since all joints remain more or less in the same vertical plane parallel to the sagittal plane.

The largest component of any joint moment by far is the one occurring about a lateral axis perpendicular to 


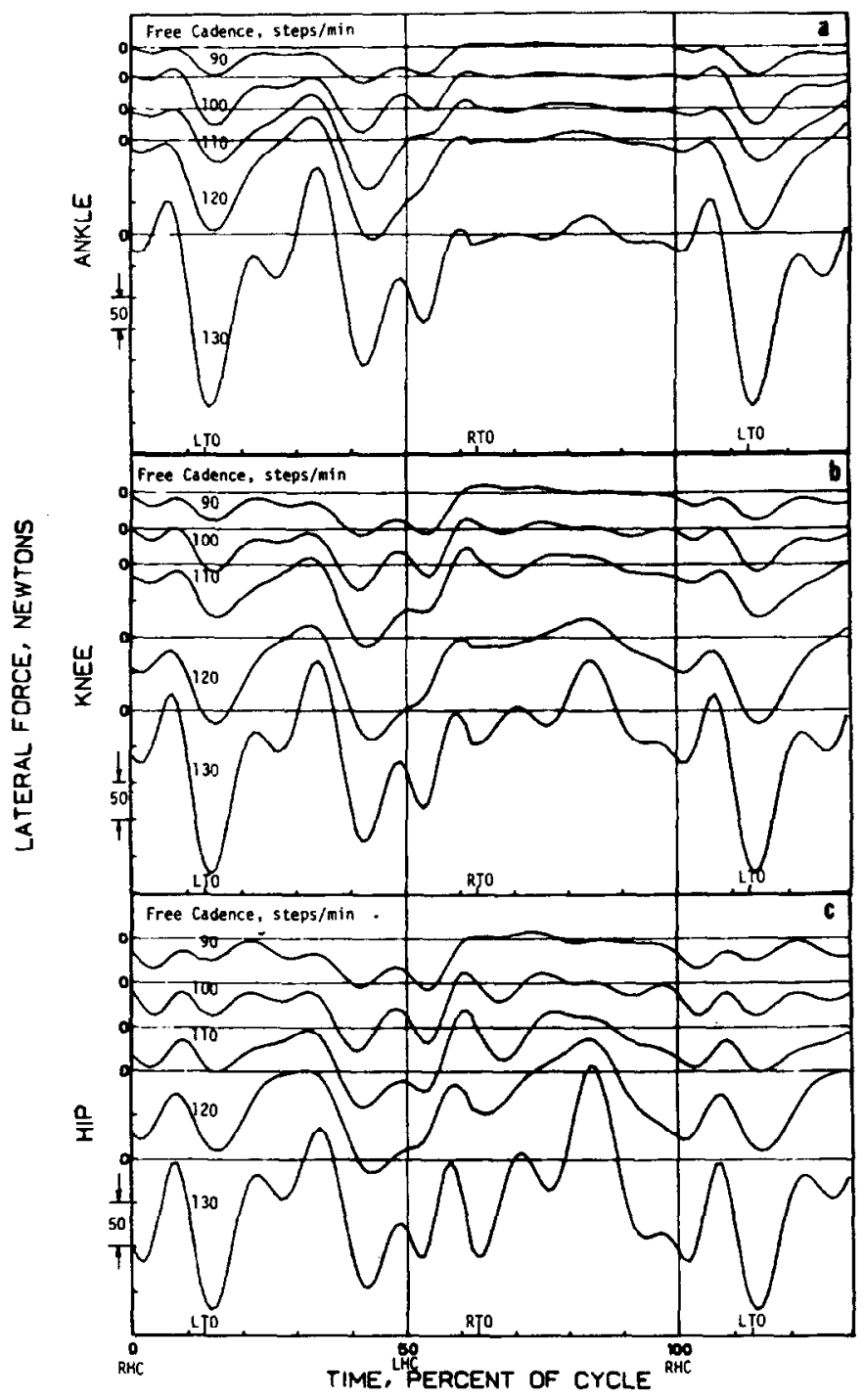

Fig. 8. Lateral components of joint forces in the right leg.

the plane of progression because it is contributed to by the two largest ground force components: vertical and forward shear. These moments are responsible for propelling the limbs and hence the body forward (Fig. 11). The ankle moments about the lateral axis (Fig. 11a) show excellent agreement with the results of Bresler and Frankel (1950). These moments are not greatly influenced by changes in step rate during much of stance phase except in early stance when a negative moment which tends to extend (plantar flex) the ankle does increase in magnitude with walking speed. The knee and hip moments in the sagittal plane are similar and generally increase in amplitude due to increased cadence. Both joints exhibit major muscular action during double support phases $(0-13$ and $50-63 \%$ of cycle). During the swing phase, minor muscular activity is present about the hip and knee, while during late swing, appreciable positive (flexor) moments are applied at both joints to decelerate the limb segments in preparation for heel contact. By contrast, the ankle moment has no significant muscle action during swing phase (Fig. 11).

\section{Power at the joints}

The main purpose of computing the moments at the joints is to permit their use in determining the power at these joints. The power output of the lower limb as a whole is the sum of the power produced at its three joints. The power supplied to the body by muscles is computed by combining the rate of work done at the joints of both lower limbs. This power is then compared to that required by the body to change the energy levels of its segments.

The power at a joint is calculated from the dot product of its moment times the relative angular 


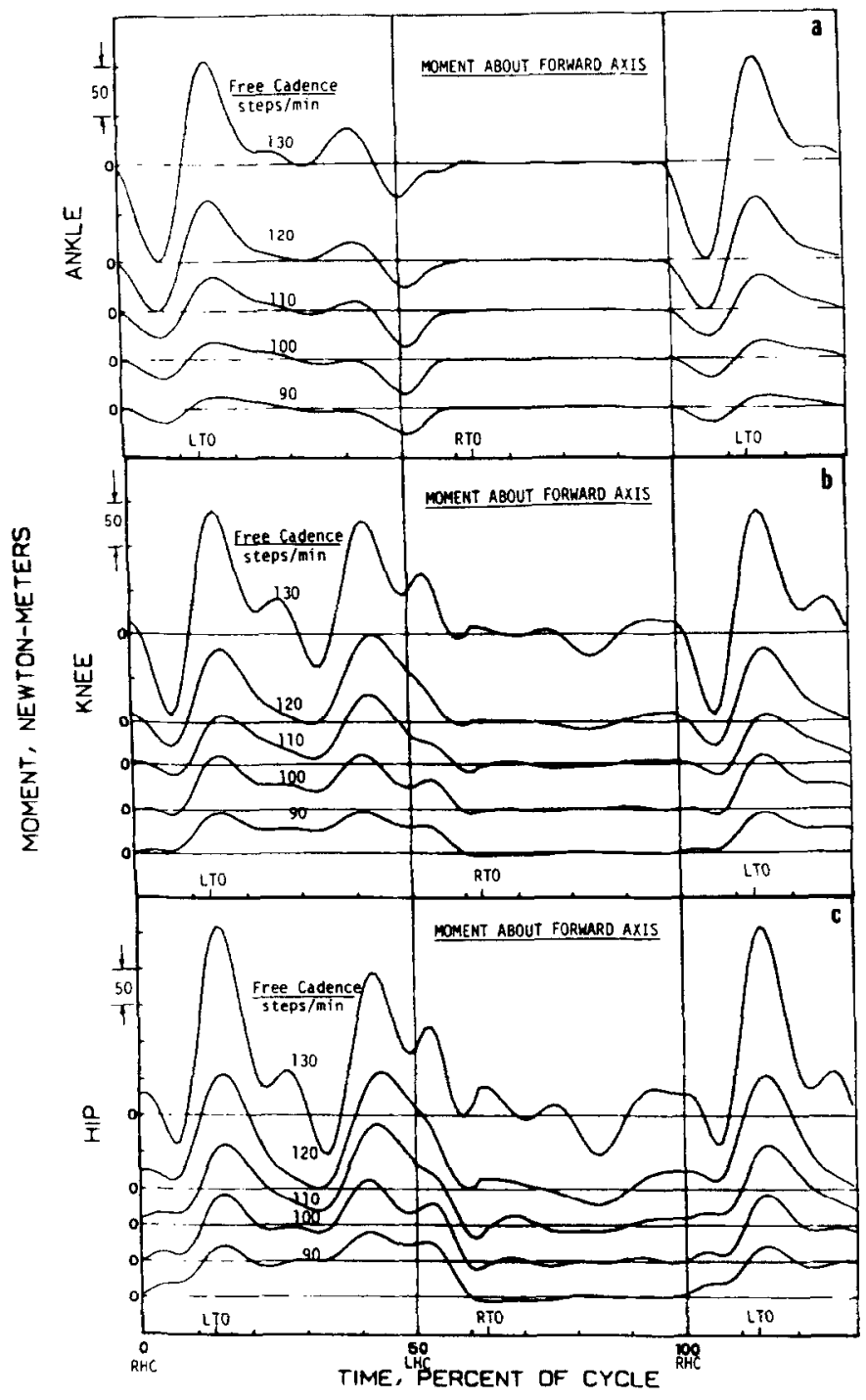

Fig. 9. Moments about a forward axis at the joints of the right leg.

velocity of the adjacent segments. Positive power or output is produced about a joint when both the moments and the angular velocity of the segment above the joint relative to the one below it act in the same direction. Negative power conditions occur when these quantities act in opposite direction. For example, the angular velocity of the thigh relative to the shank is positive during the whole double support phase. The principal knee moment component about the medial-lateral axis (Fig. 11b) is negative. Therefore, the knee joint musculature acts as a damper during this time. Figure 12 displays the power supplied or dissipated by various leg joints. These results show excellent agreement with those of Bresler and Berry (1951). Note that they displayed the flexion angles with a sign opposite to that indicated by their power curves. Both ankle and hip have outputs considerably in excess of inputs and thus constitute the principal source of power required by the body in walking. The knee joint (Fig. 12b), however, acts as a damper since it receives far more power than it produces. During late swing, additional power is dissipated by the knee to slow the shank in preparation for floor contact. Hip extension in the first half of stance phase $15-25 \%$ of cycle) requires a larger amount of power than needed by the rapid flexion occurring in double support phase (50-63\% of cycle).

The relative contributions of various joints to the power of the whole right limb are shown in Fig. 13. The power output in the lower limb is mainly contributed by the hip and ankle. This power output generally occurs in stance phase when the limb can exert large forces by pushing against the ground. During swing, the lower limb acts as a damper with most of the power dissipated at the knee (Fig. 13). The lower limb stiows additional power dissipation in early stance, reflecting 


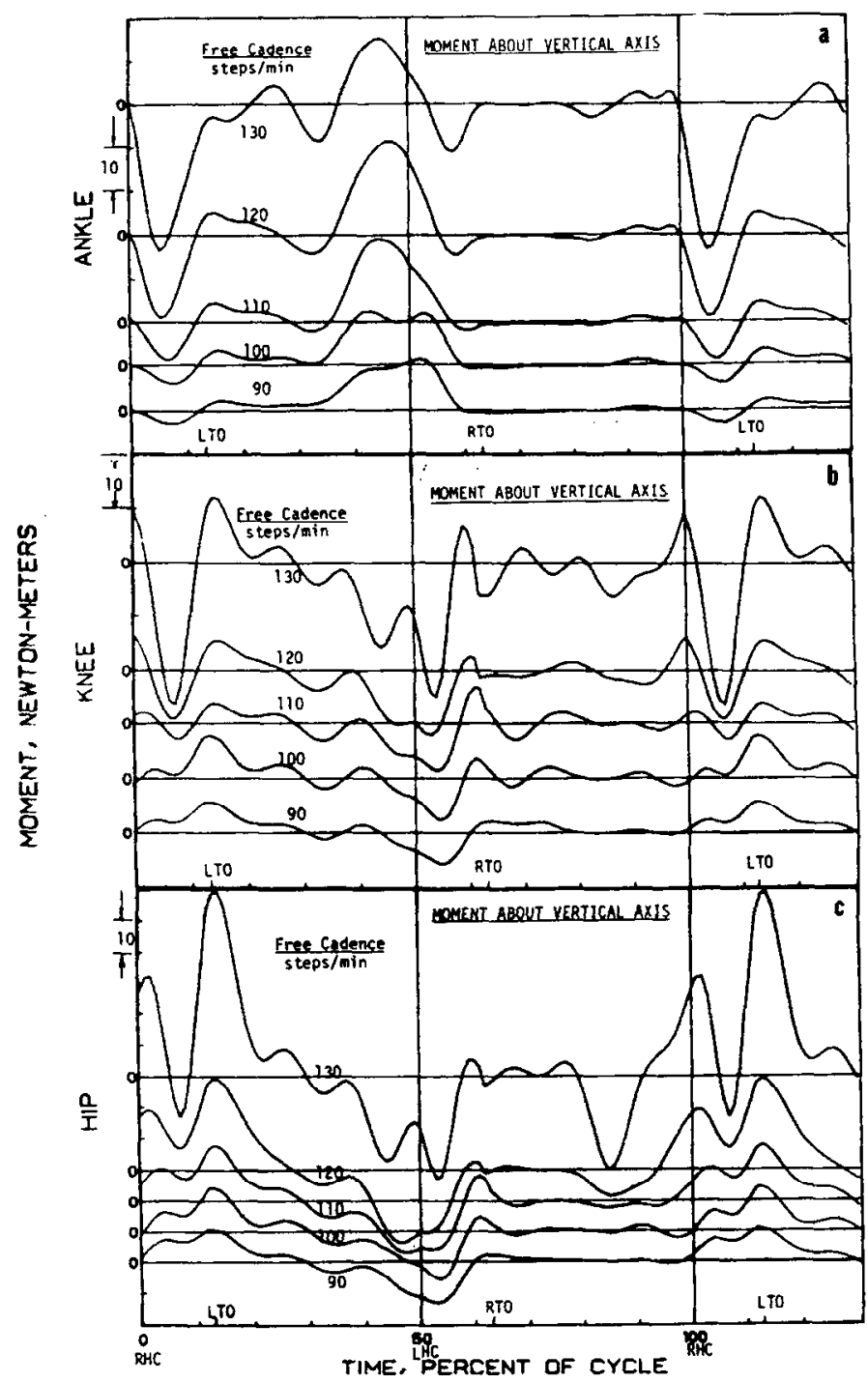

Fig. 10. Moments about a vertical axis at the joints of the right leg.

the power consumed at the knee. The power of both lower limbs is added to obtain the power supplied by the joints to the body as a whole (Fig. 14). These are compared to the power required to increase the energy level of the body as reported in Zarrugh (1981). The shapes of the curves compares well and excellent phase agreement is shown. The amplitudes do not compare as well, especially at heel contact. It is speculated that this discrepancy is due mainly to the unexpected feature in the forward shear forces of the ground.

\section{CONCLUSIONS}

1. It is feasible to predict the ground reactions on the foot during both single and double support phases, if reliable kinematic data are available and if some simple assumptions are made regarding the trajectory of the center of pressure and symmetry of forward shear forces during double support.

2. The vertical component of ground forces is the largest and has the greatest effect on the dynamics of the lower limbs in stance phase. The amplitude of the ground reactions generally increases with cadence.

3. The forces at the joints are very similar to the corresponding ground forces. This similarity decreases with height of the joint above the ground due to the increased effects of gravity and inertia, especially during the swing phase.

4. The principal components of moments at the joints are those which cause flexion or extension. The moments about a fore-and-aft axis have a magnitude intermediate between the lateral moments and moments about a vertical axis.

5. The moments at the ankle about a medial-lateral axis do not significantly change with cadence, while the hip and knee moments about the same axis increase 


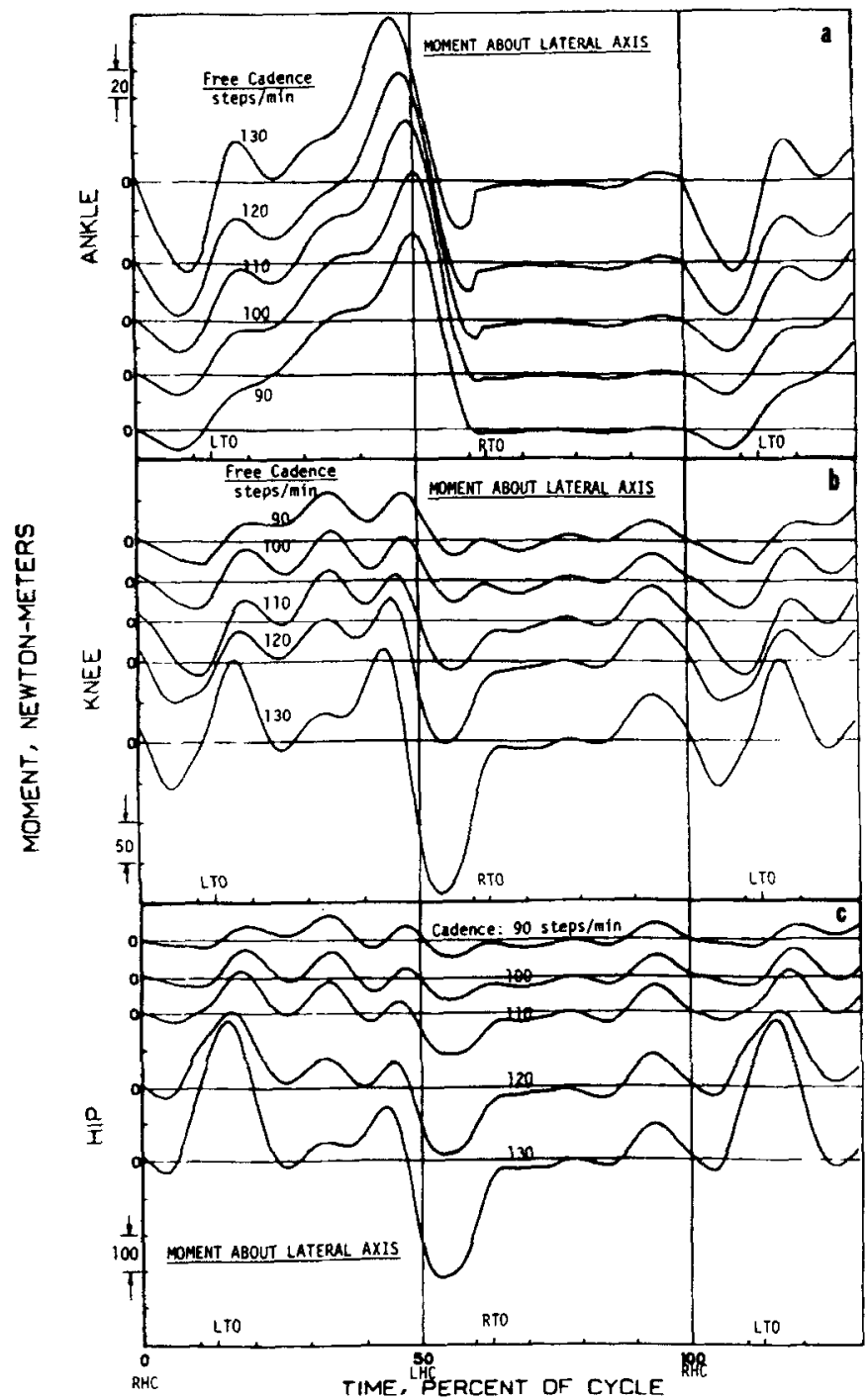

Fig. 11. Moments about a lateral axis at the joints of the right leg.

with cadence. At slow cadences, the moments at the three joints are generally of equal magnitude, while at high step rate, the hip moment has the highest maximal value followed by the knee and ankle.

6. The power generated in the lower limb is produced mainly at the hip and ankle. The knee joint generally acts as a damper. Major lower limb power exchanges occur in stance phase and particularly during the double support portion.

Acknowledgement--Research supported in part by Veterans Administration Contract VAV 101 (134).

\section{REFERENCES}

Abdelnour, T. A., Passerello, C. E. and Huston, R. L. (1975) An Analytical Analysis of Walking. ASME. Paper No. 75WA/Bio-4, ASME., New York.

Bresler, B. and Frankel, J. P. (1950) The forces and moments in the leg during normal level walking. Trans. Am. Soc. Mech. Engrs. 72, 27-36.
Bresler, B. and Berry, F. R. (1951) Energy and Power in the Leg During Level Walking. Prosthetic Devices Research Project, Institute of Engineering Research, University of California, Berkeley, Series II, Issue 15.

Cappozzo, A., Leo, T. and Pedotti, A. (1975) A general computing method for the analysis of human locomotion. J. Biomechanics. 8, 307-320.

Cunningham, D. M. (1950) Components of Floor Reactions during Walking. Prosthetic Devices Research Project, Institute of Engineering Research, University of California, Berkeley, Series II, Issue 14.

Cunningham, D. M. and Brown, G. W. (1952) Two devices for measuring the forces acting on the human body during walking. Proc. Soc. Exp. Stress Anal. 9, 75-90.

Elftman, H. (1939) Forces and energy changes in the leg during walking. Am. J. Physiol. 125, 339-356.

Elftman, H. (1940) The work done by muscles in running. $\mathrm{Am}$. J. Physiol. 129, 672-684.

Fischer, O. (1906) Theoretische Grundlagen für eine Mechanik der Lebenden Körper mit Speziellen Anwendungen auf den Menschen, sowie auf einige Bewegungs-vorgänge an Maschinen. (Theoretical Fundamentals for Mechanics of Liv- 


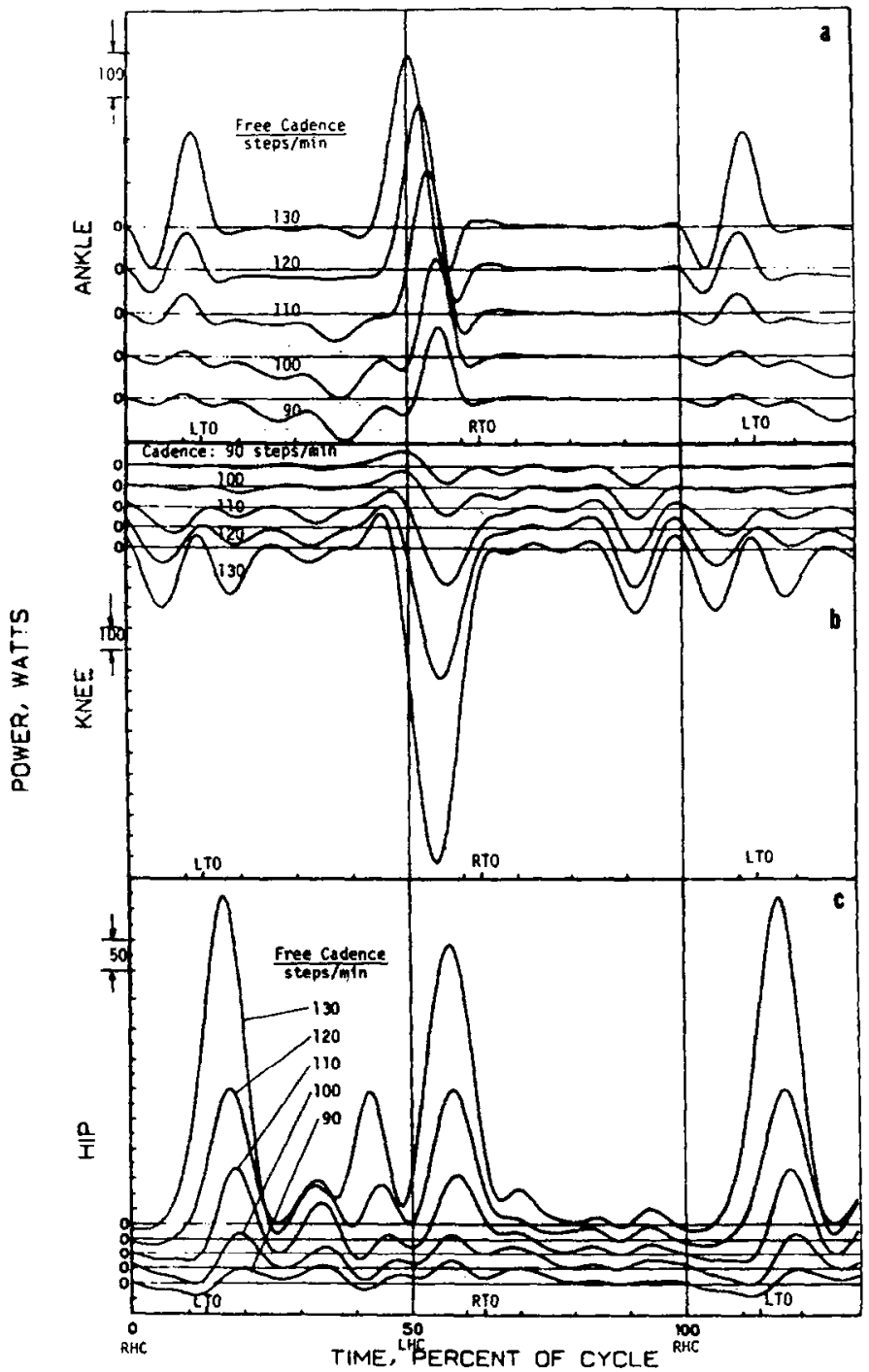

Fig. 12. Power transmitted through the joints of the right lower limb as a function of speed.

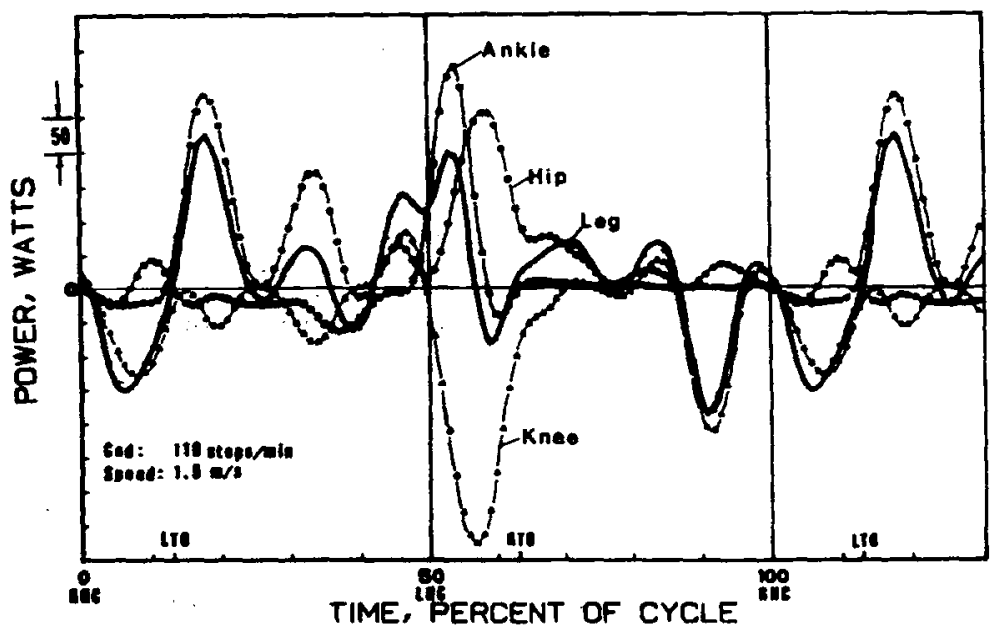

Fig. 13. Comparison of power transmitted at the joints of the right leg. 


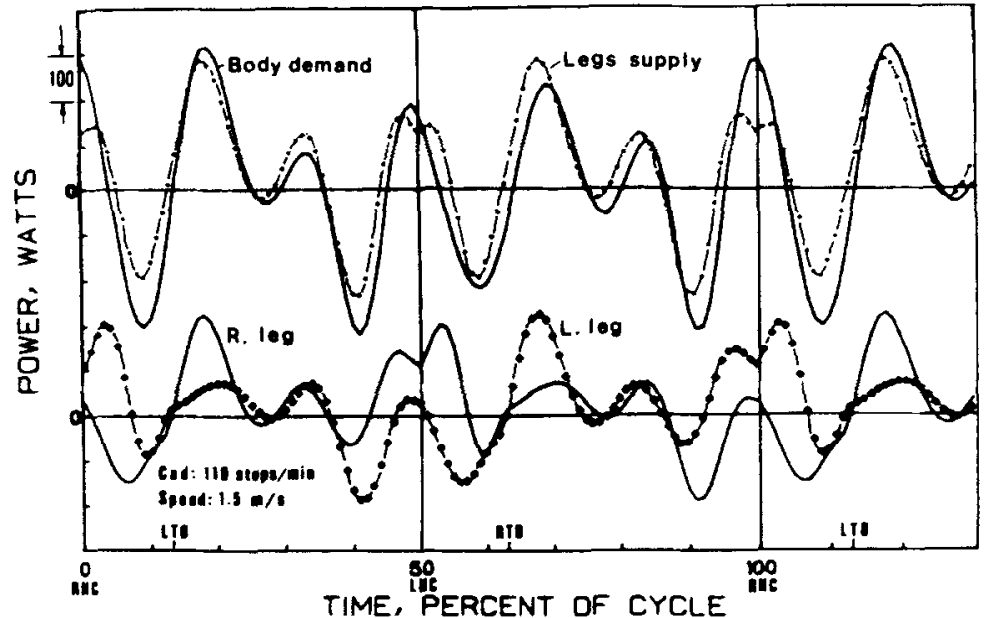

Fig. 14. Comparison between the power requirements of the body and the power supplied at the joints of the legs.

ing Bodies, with Special Applications to Man, as well as to Some Processes of Motion in Mechanics.) B. G. Teubner, Leipzig and Berlin.

McGrouther, D. A. (1974) Evaluation of a total hip replacement. J. biomed. Mater. Res. 8, 271.

Morrison, J. B. (1969) Function of the knee joint in various activities. Biomed. Engng 4, 573-580.

Paul, J. P. (1967) Forces transmitted by joints in the human body. Proc. Insin. mech. Engrs. 181(3J) 8-15.
Thorton-Trump, A. B. and Daher, R. (1975) The prediction of reaction forces from gait data. J. Biomechanics. 8, 173-178. Zarrugh, M. Y. and Radcliffe, W. W. (1979) Computer generation of human gait kinematics. J. Biomechanics 12, 99-111.

Zarrugh, M. Y. (1981) Power requirements and mechanical efficiency of treadmill walking. $J$. Biomechanics 14, 157-165. 